\title{
The Gauss-Bonnet theorem for cone manifolds and volumes of moduli spaces
}

\section{Citation}

McCullen, Curtis T. 2015. The Gauss-Bonnet theorem for cone manifolds and volumes of moduli spaces. Working Paper, Harvard University.

\section{Permanent link}

http://nrs.harvard.edu/urn-3:HUL.InstRepos:24890383

\section{Terms of Use}

This article was downloaded from Harvard University's DASH repository, and is made available under the terms and conditions applicable to Other Posted Material, as set forth at http:// nrs.harvard.edu/urn-3:HUL.InstRepos:dash.current.terms-of-use\#LAA

\section{Share Your Story}

The Harvard community has made this article openly available.

Please share how this access benefits you. Submit a story.

Accessibility 


\title{
The Gauss-Bonnet theorem for cone manifolds and volumes of moduli spaces
}

\author{
Curtis T. McMullen*
}

17 June 2013

\begin{abstract}
This paper generalizes the Gauss-Bonnet formula to a class of stratified spaces called Riemannian cone manifolds. As an application, we compute the volumes of the moduli spaces $\mathcal{M}_{0, n}$ with respect to the complex hyperbolic metrics introduced by Picard, Deligne-Mostow and Thurston.
\end{abstract}

\section{Contents}

1 Introduction . . . . . . . . . . . . . . . . . . 1

2 Polyhedra...................... 4

3 Spherical complexes .................. 8

4 Riemannian complexes ................. . 12

5 Cone manifolds . . . . . . . . . . . . . . . . . . . . 15

6 Euler characteristics of strata . . . . . . . . . . . . . 19

7 Gauss-Bonnet for cone manifolds . . . . . . . . . . . . . . 23

8 Volumes of moduli spaces . . . . . . . . . . . . . . . 24

9 Hyperbolic twists and non-arithmetic groups . . . . . . . . 28

\section{Introduction}

The Gauss-Bonnet formula for a closed Riemannian manifold states that the Euler characteristic $\chi(M)$ is given by a curvature integral, $\int_{M} \Psi(x) d v(x)$. Here we generalize this formula to compact Riemannian cone manifolds.

By definition, an $n$-dimensional cone manifold $M$ is locally isometric to the cone over an $(n-1)$-dimensional spherical cone manifold $K(\S 5)$. The cone can be built using any Riemannian metric on the unit ball $B^{n}$ that is

\footnotetext{
${ }^{*}$ Research supported in part by the NSF. 2000 Mathematics Subject Classification: 53C20, 32G15. Revised 24 April 2015.
} 
invariant under the holonomy of $K$; while $K$ itself can be assembled from spherical simplices by gluing their faces together in pairs.

A cone manifold is naturally partitioned into connected strata $M^{\sigma}$, each of which is a totally geodesic Riemannian manifold. The solid angle of $M$ at $x$, defined by

$$
\Theta(x)=\lim _{r \rightarrow 0} \frac{\operatorname{vol}_{n}(B(x, r))}{\operatorname{vol}_{n}\left(B^{n}\right) r^{n}},
$$

is a constant along each stratum; its value on $M^{\sigma}$ will be denoted by $\Theta^{\sigma}$.

Let $M[n]$ denote the union of top-dimensional strata of $M$. In $\S 7$ we will show:

Theorem 1.1 A compact cone manifold of dimension $n$ satisfies

$$
\int_{M[n]} \Psi(x) d v(x)=\sum_{\sigma} \chi\left(M^{\sigma}\right) \Theta^{\sigma} .
$$

For a smooth manifold the right-hand side reduces to $\chi(M)$ and we obtain the usual Gauss-Bonnet formula. For orbifolds the right-hand terms have rational weights of the form $\Theta^{\sigma}=1 /\left|H^{\sigma}\right|$, and we obtain Satake's formula [Sat]. In a cone manifold $\Theta^{\sigma}$ can assume any positive real value, and the right-hand side provides a natural generalization of the orbifold Euler characteristic of $M$.

Moduli spaces. Let $\mathcal{M}_{0, n}$ denote the moduli space of configurations of $n \geq 3$ ordered points on the Riemann sphere. Generalizing work of Picard and Deligne-Mostow, Thurston showed that for any set of real weights $\left(\mu_{1}, \ldots, \mu_{n}\right)$ with $0<\mu_{i}<1$ and $\sum \mu_{i}=2$, we have a natural complex hyperbolic metric $g_{\mu}$ on $\mathcal{M}_{0, n}$, and its metric completion $P(\mu)$ is a cone manifold [Th]. (The notation $P(\mu)$ is a reminder that the $n$ points are ordered, so their monodromy produces pure braids.)

Applying Theorem 1.1, in $\S 8$ we will show:

Theorem 1.2 The complex hyperbolic volume of moduli space satisfies

$$
\operatorname{vol}\left(\mathcal{M}_{0, n}, g_{\mu}\right)=C_{n-3} \sum_{\mathcal{P}}(-1)^{|\mathcal{P}|+1}(|\mathcal{P}|-3) ! \prod_{B \in \mathcal{P}} \max \left(0,1-\sum_{i \in B} \mu_{i}\right)^{|B|-1}
$$

Here $\mathcal{P}$ ranges over all partitions of $\{1, \ldots, n\}$ into blocks $B$, and $C_{k}=$ $(-4 \pi)^{k} /(k+1)$ ! is the value of $\Psi(x)$ on a complex hyperbolic $k$-manifold. Previously the volume has been computed only in special cases [Yo], [Sau], [Par]. 
Orbifolds and volume ratios. There are 94 values of $\mu$ with $n \geq 5$ such that a natural finite quotient $M(\mu)$ of $P(\mu)$ is a complex hyperbolic orbifold. Their Euler characteristics are tabulated in $\S 8$, and their volumes can be obtained by multiplying by $C_{n-3}$.

Deligne and Mostow observed that 16 of these orbifolds yield non-arithmetic lattices $\Gamma(\mu)$ in $\mathrm{PU}(1, n)$. In an early version of $[\mathrm{KM}]$, Kappes and Möller showed that the Lyapunov exponents attached to these lattices and their Galois conjugates are useful commensurability invariants. Motivated by their striking result, we proposed the volume ratio

$$
\rho(\mu, \nu)=\frac{\operatorname{vol}(P(\nu))}{\operatorname{vol}(P(\mu))}=\frac{\chi(P(\nu))}{\chi(P(\mu))}
$$

between $P(\mu)$ and its hyperbolic twists $P(\nu)$ as another invariant, which is easily computed using Theorem 1.2. The values of this ratio turn out to distinguish the commensurability classes of all but one of the non-arithmetic Deligne-Mostow examples ( $\$ 9)$.

Polyhedra and cell complexes. We give a self-contained account of the theory of cone manifolds, relying on a 'unique factorization theorem' in spherical geometry, in $\S 5$. Thurston's $(X, G)$ cone manifolds are a special case of those considered here.

Our proof of Theorem 1.1 is based on the Gauss-Bonnet formula for Riemannian polyhedra ( $(2)$, proved in the 1940s by Allendoerfer and Weil. To apply this formula to cone manifolds, two main challenges must be addressed.

The first is that the formula for polyhedra is given in terms of outer angles, instead of inner angles. We relate the two types of angles in Theorem 3.1, which is obtained by comparing a spherical complex $M$ with its suspension $S^{0} * M$. As a corollary we obtain a Gauss-Bonnet formula for cell complexes with totally geodesic faces (Theorem 4.1), which states that

$$
\sum_{|\alpha|=\operatorname{dim}(M)} \int_{M_{\alpha}} \Psi(x) d v(x)=\sum_{\alpha}(-1)^{|\alpha|} \Theta_{\alpha} .
$$

The second is that, when one subdivides a cone manifold into polyhedra, it may be necessary to fracture its totally geodesic strata into pieces with curved faces. These faces introduce complicated expressions involving the second fundamental form, which must be shown to cancel. This cancellation is derived from invariance of Weyl's tube formula and properties of the spherical dual of a suspension $(\S 6)$. 
Notes and references. Cone manifolds and related spaces appear frequently in differential geometry, especially in the special case of singularities along a codimension two submanifold; see e.g. [Ba2], [Cg], [HMM], [FST], $[\mathrm{Ko} 1],[\mathrm{CHK}],[\mathrm{Br}],[\mathrm{Tr}]$ and $[\mathrm{AB}]$. A general account of the theory of stratified spaces is given in $[\mathrm{Pf}]$.

The first proof of the Gauss-Bonnet theorem for general manifolds was given by Allendoerfer and Weil [AW], using Weyl's tube formula [We]. Chern's intrinsic proof $[\mathrm{Cn}]$ appeared shortly thereafter. The tube formula and its applications in differential geometry are treated in $[\mathrm{Gr}]$. Theorem 1.1 can be extended to cone manifolds with curved strata by using the full strength of the formula for polyhedra.

The volume of $\mathcal{M}_{0, n}$ with respect to its natural symplectic structure is computed in $[\mathrm{Zo}]$. The Euler characteristic of the Deligne-Mumford compactification $\overline{\mathcal{M}_{0, n}}$ can be computed using inversion of powers series; see [Ge], [Mc3]. The compactifications $M(\mu)$ resulting from a choice of weights are usual different from $\overline{\mathcal{M}_{0, n}}$, and depend on $\mu$; they are discussed from the perspective of algebraic geometry in [Ha]. Volumes of arithmetic lattices in $\mathrm{SU}(n, 1)$ are discussed in the recent paper [ES].

Acknowledgements. I would like to thank Kappes and Möller for useful correspondence.

\section{Polyhedra}

The results from $[\mathrm{AW}]$ we will need are summarized in this section and in Proposition 3.2 of the next.

We begin with the Gauss-Bonnet formula for polyhedra, which may be stated as follows.

Theorem 2.1 (Allendoerfer-Weil) The Euler characteristic of a compact Riemannian polyhedron $M$ of dimension $n$ satisfies

$$
(-1)^{n} \chi^{\prime}(M)=\int_{M[n]} \Psi(x) d v(x)+\sum_{r=0}^{n-1} \int_{M[r]} d v(x) \int_{N(x)^{*}} \Psi(x, \xi) d \xi .
$$

Here $\chi^{\prime}(M)=\chi(M)-\chi(\partial M)$.

In this section we explain the formula above and the basic properties of its integrand. 
Spheres and duality. Let $S^{n}$ denote the unit sphere in $\mathbb{R}^{n+1}$, and let $B^{n+1}$ denote the unit ball. We let

$$
\omega_{n}=2 \cdot \frac{(4 \pi)^{n / 2}(n / 2) !}{n !}
$$

denote the volume of $S^{n}$, with the convention that $(n / 2) !=\Gamma\left(\frac{n}{2}+1\right)$ if $n$ is odd.

A convex spherical polyhedron is a set $K \subset S^{n}$ obtained by intersecting finitely many hemispheres. Its dual is given by

$$
K^{*}=\left\{x \in S^{n}:\langle x, y\rangle \geq 0 \forall y \in K\right\} .
$$

When the ambient sphere needs to be emphasized, we will write $K^{*}=$ $\left(K, S^{n}\right)^{*}$.

Manifolds. Let $A$ be a smooth Riemannian manifold of dimension $n$. When $n$ is even, the intrinsic Gauss-Bonnet integrand on $A$ is given in terms of the Riemann curvature tensor by

$$
\Psi(x)=\frac{2}{\omega_{n}} \cdot \frac{1}{2^{n / 2} n !} \sum_{i, j \in S_{n}} \frac{\epsilon(i) \epsilon(j)}{g} R_{i_{1} i_{2} j_{1} j_{2}} \cdots R_{i_{n-1} i_{n} j_{n-1} j_{n}} .
$$

Here $\epsilon(i)$ and $\epsilon(j)$ denote the signs of the permutations $i$ and $j$, and $g$ is the determinant of the metric. When $n$ is odd, we set $\Psi(x)=0$. The Gauss-Bonnet theorem states that for any closed manifold $A$ we have

$$
\chi(A)=\int_{A} \Psi(x) d v(x) .
$$

Submanifolds. Now let $A$ be an $r$-dimensional submanifold of a Riemannian manifold $B$ of dimension $n$. Let $R_{i j k l}$ denote the restriction of the Riemann curvature tensor on $B$ to $A$, and let $\Lambda_{i j}(\xi)$ denote the second fundamental form - a symmetric tensor on $A$ depending linearly on a normal vector $\xi$. In local coordinates where $A \subset B$ is modeled on $\mathbb{R}^{r} \subset \mathbb{R}^{n}$, we have

$$
\Lambda_{i j}(\xi)=\left\langle\nabla_{e_{i}} e_{j}, \xi\right\rangle \text {. }
$$

The extrinsic Gauss-Bonnet integrand is the function on the unit normal bundle to $A$ defined by

$$
\begin{aligned}
\Psi(x, \xi)= & \sum_{0 \leq 2 f \leq r} \Psi_{r, f}(x, \xi), \text { where } \\
\Psi_{r, f}(x, \xi)= & \frac{2}{\omega_{2 f} \omega_{n-2 f-1}} \cdot \frac{1}{2^{f}(2 f) !(r-2 f) !} \cdot \sum_{i, j \in S_{r}} \frac{\epsilon(i) \epsilon(j)}{\gamma} \times \\
& R_{i_{1} i_{2} j_{1} j_{2}} \cdots R_{i_{2 f-1} i_{2 f} j_{2 f-1} j_{2 f}} \Lambda_{i_{2 f+1} j_{2 f+1}}(\xi) \cdots \Lambda_{i_{r} j_{r}}(\xi) .
\end{aligned}
$$


Here $\gamma$ is the determinant of the induced metric on $A$.

The intrinsic and extrinsic integrands on $A$ are related by

$$
\Psi(x)=\int_{S(x)} \Psi(x, \xi) d \xi
$$

where $S(x)$ is the unit sphere (of dimension $n-r-1$ ) in the normal bundle to $A$ at $x$, and $\int_{S(x)} d \xi=\omega_{n-r-1}$. In particular, if $A$ is a closed submanifold of $B$ then we have

$$
\chi(A)=\int_{A} d v(x) \int_{S(x)} \Psi(x, \xi) d \xi
$$

Example. Suppose $r$ is even, $n>r$ and

$$
A=S^{2 f} \times S^{r-2 f} \subset S^{2 f} \times \mathbb{R}^{n-2 f}=B .
$$

Then $\Psi(x, \xi)=\Psi_{r, f}(x, \xi)$. In a standard orthonormal frame there are $2^{f}(2 f) !(r-2 f)$ ! nonzero terms in the formula for $\Psi_{r, f}(x, \xi)$, since only permutations in $S_{r}$ which preserve the indices $(1,2, \ldots, 2 f)$ contribute, and we have $R_{a b c d}=g_{a c} g_{b d}-g_{a d} g_{b c}$ on the unit sphere. Since the second fundamental form $\Lambda_{i j}(\xi)$ depends only on the projection of $\xi$ to a 1-dimensional space, the integral of each nonzero term is given by

$$
\begin{aligned}
\operatorname{vol}_{r}(A) \int_{S^{n-r-1}}\left|\xi_{1}\right|^{r-2 f} d \xi & =\omega_{2 f} \int_{S^{n-r-1}} \omega_{r-2 f}\left|\xi_{1}\right|^{r-2 f} d \xi \\
& =2 \omega_{2 f} \omega_{n-2 f-1}
\end{aligned}
$$

The product of this constant with the number of nonzero terms cancels with the leading constant factor in the formula for $\Psi_{r, f}(x, \xi)$, yielding the expected result $\chi(A)=4$.

Basic relation. More generally, consider a pair of smoothly embedded submanifolds $A \subset B \subset C$. Let $S_{B}(x)$ and $S_{C}(x)$ denote the fiber of the unit normal bundle to $A$ at $x$, where $A$ is considered as a submanifold of $B$ and $C$ respectively. A convex set $K \subset S_{B}(x) \subset S_{C}(x)$ then has two duals, namely $K_{B}^{*}=\left(K, S_{B}(x)\right)^{*}$ and $K_{C}^{*}=\left(K, S_{C}(x)\right)^{*}$. Similarly, the manifold $A$ has two extrinsic Gauss-Bonnet integrands, one for $B$ and one for $C$. We then have the basic relation:

$$
\int_{K_{B}^{*}} \Psi_{B}(x, \xi) d \xi=\int_{K_{C}^{*}} \Psi_{C}(x, \xi) d \xi .
$$

Equation (2.1) can be regarded as a special case of (2.2), with $A=B$ and $K=\emptyset$. For proofs of these relations, see [AW, (6), (7), p.121]. 
Polyhedra. A convex Euclidean polyhedron is a set $K \subset \mathbb{R}^{n}$ obtained by intersecting finitely many half-spaces. A smooth polyhedron is a connected Hausdorff space $M$ equipped with local charts taking values in convex Euclidean polyhedra, such that the transition maps between charts are smooth.

When $M$ is endowed with a smooth metric, it becomes a Riemannian polyhedron $[\mathrm{AW}]$.

The inward pointing normals $\boldsymbol{N}(\boldsymbol{x})$. Given an $n$-dimensional Riemannian polyhedron and $r<n$, we let $M[r]$ denote the union of the $r$ dimensional faces of $\partial M$, and let $M[n]=M-\partial M$. Each face of $M$ can be regarded as a submanifold of a slight thickening of $M[n]$, so its normal bundle and its extrinsic Gauss-Bonnet integrand are defined. Given $x \in M[r]$, $r<n$, we let

$$
N(x) \subset S(x)
$$

denote the convex set of unit vectors normal to $M[r]$ that point into $M[n]$.

Sketch of the proof of Gauss-Bonnet. These definitions complete the statement of the Gauss-Bonnet formula for polyhedra (Theorem 2.1). We conclude with a sketch of the proof; for details, see [AW, Theorem II].

First suppose $M$ is a simplex. Choose an isometric embedding $M \hookrightarrow$ $\mathbb{R}^{N+1}$ for some large $N$. Let $T \subset \mathbb{R}^{N+1}$ be the boundary of a small tube around the image, i.e. the set of points at distance $\delta>0$ from $M$. Let $\gamma: T \rightarrow S^{N}$ denote the Gauss map. For $\delta$ sufficiently small, we also have a nearest-point projection $\pi: T \rightarrow M$.

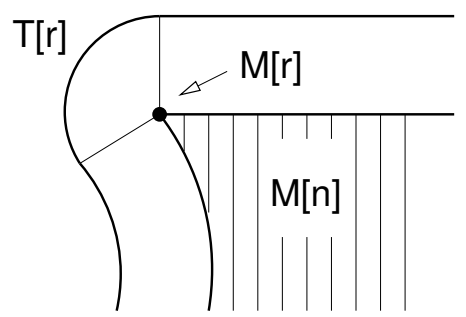

Figure 1. The tube around a polyhedron in $\mathbb{R}^{N+1}$.

Let $T[r]=\pi^{-1}(M[r])$ be the subset of $T$ closest to $M[r]$. Then for any $t \in T[r]$ we have a vector $\xi=\gamma(t) \in S^{N}$ and a point $x=\pi(t) \in M[r]$. The pair $(x, \xi)$ satisfies $\langle\xi, v\rangle=0$ for all $v \in T_{x} M[r]$, and

$$
\langle\xi, v\rangle \leq 0 \quad \forall v \in N(x) \subset T_{x} \mathbb{R}^{N+1} .
$$

Conversely, if $(x, \xi)$ satisfies the conditions above, then there is unique $t \in T$ with $\pi(t)=x$ and $\gamma(t)=v$. Using this fact and Weyl's tube formula [We], 
one finds that the contributions to the degree of the Gauss map are given by

$$
\mathcal{G}(M[n])=\frac{1}{\omega_{N}} \int_{T[n]} \gamma^{*}(d \xi)=\int_{M[n]} \Psi(x) d v(x)
$$

and for $r<n$,

$$
\mathcal{G}(M[r])=\frac{1}{\omega_{N}} \int_{T[r]} \gamma^{*}(d \xi)=\int_{M[r]} d v(x) \int_{N(x)^{*}} \Psi(x, \xi) d \xi
$$

(Remarkably, these quantities are independent of the choice of isometric embedding $M \hookrightarrow \mathbb{R}^{N+1}$.) Since $M$ is a simplex, the Gauss map has degree one, and hence $\sum_{r=0}^{n} \mathcal{G}(M[r])=1=(-1)^{n} \chi^{\prime}(M)$ as desired.

For the general case Allendoerfer and Weil rely on a local isometric embedding theorem for analytic metrics due to Cartan, since the Nash embedding theorem was not available at the time. They first establish Theorem 2.1 for the simplices in a fine subdivision of $M$, and then use an addition formula based on (2.1) and (2.2) to deduce the Gauss-Bonnet theorem for $M$ itself. Relations (2.1) and (2.2) are proved using invariance of the tube formula under different isometric embeddings.

\section{Spherical complexes}

In this section we define spherical complexes, which provide models for the unit tangent bundle in the setting of singular spaces. We then discuss the inner and outer angles $\theta_{\alpha}$ and $\Omega_{\alpha}$ of their cells $M_{\alpha}$, and show:

Theorem 3.1 The outer angle of a compact spherical complex $M$ is given by

$$
\Omega(M)=\sum_{\alpha} \theta_{\alpha}\left(1-\Omega_{\alpha}\right)
$$

Spherical complexes. A cell complex is a Hausdorff topological space $M$ equipped with a partition into disjoint cells $M_{\alpha}$ such that the following conditions hold:

1. The closure of each $n$-cell is homeomorphic to a closed ball in $\mathbb{R}^{n}$, with the cell itself corresponding to the interior of the ball.

2. Whenever $M_{\alpha}$ meets $\overline{M_{\beta}}$ we have $M_{\alpha} \subset \overline{M_{\beta}}$. 
A 0 -cell is simply a point. We denote the dimension of $M_{\alpha}$ by $|\alpha|$, and we write $\alpha \leq \beta$ if $M_{\alpha} \subset \overline{M_{\beta}}$.

A spherical complex is a cell complex built out of spherical polyhedra, in such a way that the inclusions $M_{\alpha} \subset \overline{M_{\beta}}$ are isometries. We also require that each face of $\overline{M_{\beta}}$ is a cell; equivalently, that for $r<|\beta|$ we have

$$
\overline{M_{\beta}}[r]=\bigcup\left\{M_{\alpha}: \alpha \leq \beta,|\alpha|=r\right\} .
$$

Outer angles of polyhedra. The normalized outer angle of a convex polyhedron $K \subset S^{n}$ is given by

$$
\omega(K)=\frac{\operatorname{vol}\left(K^{*}\right)}{\operatorname{vol}\left(S^{n}\right)} .
$$

It is easily verified that this quantity is invariant under the inclusion $S^{n} \subset$ $S^{n+1}$; thus it depends only on $K$, not on the ambient sphere. The outer angle measures the proportion of $S^{n}$ that is closer to the vertex of the cone $\mathbb{R}_{+} \cdot K$ than to any other point of $\mathbb{R}_{+} \cdot K$; thus it measures the size of $T[r]$ in Figure 1, explaining its relation to the tube formula.

Outer angles of complexes. The outer angle of a compact spherical complex is defined by:

$$
\Omega(M)=\sum_{\alpha}(-1)^{|\alpha|} \omega\left(M_{\alpha}\right)
$$

If two spherical complexes $M$ and $M^{\prime}$ have isomorphic subdivisions, then $\Omega(M)=\Omega\left(M^{\prime}\right)$. More precisely, we have:

Proposition 3.2 (Allendoerfer-Weil) Let $K \subset S^{n}$ be an open convex polyhedron, subdivided into convex cells $\left(K_{\alpha}\right)$. Then

$$
(-1)^{n} K^{*}=\sum(-1)^{|\alpha|} K_{\alpha}^{*}
$$

as $n$-chains on $S^{n}$.

See $\left[\mathrm{AW}\right.$, Theorem 3]. The equality of chains means $(-1)^{n} \int_{K^{*}} f d \xi=$ $\sum_{\alpha}(-1)^{|\alpha|} \int_{K_{\alpha}^{*}} f d \xi$ for any $f \in C\left(S^{n}\right)$. Taking $f=1$, we obtain $(-1)^{n} \omega(K)=$ $\sum(-1)^{|\alpha|} \omega\left(K_{\alpha}\right)$, which implies that $\Omega(M)$ is invariant under subdivision.

Random Euler characteristic. We note that if $M$ is isometrically embedded in $S^{n}$ then its outer angle is related to the expected Euler characteristic of its intersection with a random spherical hyperplane $H \cong S^{n-1}$ : we have

$$
2 \Omega(M)=\chi(M)+\mathbb{E}(\chi(H \cap M)) .
$$


The unit normal complex. Given $x \in M_{\alpha}$ and $\beta>\alpha$, we let

$$
S_{\alpha \beta}(x)=\left\{v \in T_{x} \overline{M_{\beta}}: v \text { is a unit normal to } M_{\alpha}\right\}
$$

denote the unit tangent sphere (of dimension $|\beta|-|\alpha|-1$ ) normal to $M_{\alpha}$. The directions pointing into $M_{\beta}$ determine an open, convex polyhedron

$$
N_{\alpha \beta}(x) \subset S_{\alpha \beta}(x)
$$

called the inner angle. These spherical polyhedra can be assembled to form a spherical complex

$$
N_{\alpha}(x)=\bigcup_{\beta>\alpha} N_{\alpha \beta}(x)
$$

called the unit normal complex for $M_{\alpha}$ at $x$.

Angles. The normalized volume of the cell $M_{\alpha}$ and the normalized inner and outer angles of the cells $N_{\alpha \beta}(x)$ will be denoted by

$$
\begin{aligned}
\theta_{\alpha} & =\operatorname{vol}\left(M_{\alpha}\right) / \operatorname{vol}\left(S^{|\alpha|}\right), \\
\theta_{\alpha \beta}(x) & =\operatorname{vol}\left(N_{\alpha \beta}(x)\right) / \operatorname{vol}\left(S_{\alpha \beta}(x)\right) \text { and } \\
\omega_{\alpha \beta}(x) & =\operatorname{vol}\left(N_{\alpha \beta}(x)^{*}\right) / \operatorname{vol}\left(S_{\alpha \beta}(x)\right) .
\end{aligned}
$$

Parallel transport along a geodesic provides an isometric map from $N_{\alpha \beta}(x)$ to $N_{\alpha \beta}(y)$ for any $x, y \in M_{\alpha}$, so in fact the angles above are independent of $x$. They will therefore be denoted simply by $\theta_{\alpha \beta}$ and $\omega_{\alpha \beta}$. Because they are constant, and $\operatorname{dim} N_{\alpha \beta}(x)=|\beta|-|\alpha|-1$, the outer angle $\Omega\left(N_{\alpha}(x)\right)$ is given by

$$
\Omega_{\alpha}=(-1)^{|\alpha|+1} \sum_{\beta>\alpha}(-1)^{|\beta|} \omega_{\alpha \beta}
$$

for all $x \in M_{\alpha}$. In particular, $\Omega_{\alpha}=0$ if $|\alpha|=\operatorname{dim}(M)$.

An index $\alpha$ is even if $|\alpha| \equiv 0 \bmod 2$.

Theorem 3.3 The Euler characteristic of a compact spherical complex satisfies

$$
\chi(M)=2 \sum_{\text {even } \alpha} \theta_{\alpha}\left(1-\Omega_{\alpha}\right) .
$$

Proof. Clearly $\chi(M)=\sum_{\beta}(-1)^{|\beta|}=\sum_{\beta} \chi^{\prime}\left(\overline{M_{\beta}}\right)$. By the Gauss-Bonnet formula from Theorem 2.1, we have

$$
(-1)^{|\beta|} \chi^{\prime}\left(\overline{M_{\beta}}\right)=\int_{M_{\beta}} \Psi(x) d v(x)+\sum_{\alpha<\beta} \int_{M_{\alpha}} d v(x) \int_{N_{\alpha \beta}(x)^{*}} \Psi(x, \xi) d \xi .
$$


Here we have used equation (3.1) to convert the integral over $M[r]$ into an integral over cells. Since $M_{\beta}$ is a spherical polyhedron of dimension $n=|\beta|$, we have $\Psi(x)=\left(1 / \omega_{n}\right)\left(1+(-1)^{n}\right)$. Since the spherical polyhedron $M_{\alpha}$ is totally geodesic, its second fundamental form vanishes and the curvature tensors on $M_{\beta}$ and $M_{\alpha}$ agree. Thus $\Psi(x, \xi)=\left(1 / \omega_{r}\right)\left(1 / \omega_{n-r-1}\right)\left(1+(-1)^{r}\right)$, where $r=|\alpha|$. Integrating, we obtain

$$
\chi^{\prime}\left(\overline{M_{\beta}}\right)=\theta_{\beta}\left(1+(-1)^{|\beta|}\right)+(-1)^{|\beta|} \sum_{\alpha<\beta} \theta_{\alpha}\left(1+(-1)^{|\alpha|}\right) \omega_{\alpha \beta} .
$$

Summing over $\beta$ and then rearranging terms, we obtain

$$
\chi(M)=2 \sum_{\text {even } \alpha} \theta_{\alpha}\left(1+\sum_{\beta>\alpha}(-1)^{|\beta|} \omega_{\alpha \beta}\right) .
$$

The stated formula now follows from equation (3.3).

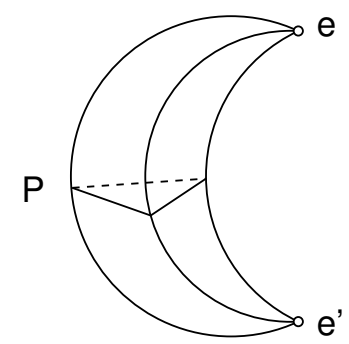

Figure 2. The suspension $S^{0} * P$ of the cell $P$.

Suspensions. Let $P$ be a convex polyhedron in $S^{n}$, considered as the equator of $S^{n+1}$, and let $\left\{e, e^{\prime}\right\} \cong S^{0}$ be the corresponding pair of poles. The suspension $S^{0} * P$ is defined by taking the convex hull of $P \cup\left\{e, e^{\prime}\right\}$, as in Figure 2; it is swept out by the geodesic arcs joining the poles that pass through $P$, and satisfies

$$
\frac{\operatorname{vol}\left(S^{0} * P\right)}{\operatorname{vol}\left(S^{n+1}\right)}=\frac{\operatorname{vol}(P)}{\operatorname{vol}\left(S^{n}\right)}
$$

Proof of Theorem 3.1. Let $S^{0} * M$ denote the compact spherical complex assembled from the cells $S^{0} * M_{\alpha}$, together with the two additional points $e$ and $e^{\prime}$. By Theorem 3.3, we have

$$
\chi(M)=2 \sum_{\text {even } \alpha} \theta_{\alpha}\left(1-\Omega_{\alpha}\right) .
$$


Suspension of $M_{\alpha}$ does not change the value of $\theta_{\alpha}$ or of $\Omega^{\alpha}$, but it does shift its dimension by one. To construct $S^{0} * M$ we must also add two new points, $e$ and $e^{\prime}$, each with outer angle $\Omega(M)$. By Theorem 3.3 again, we then have

$$
\chi\left(S^{0} * M\right)=2 \sum_{\text {odd } \alpha} \theta_{\alpha}\left(1-\Omega_{\alpha}\right)+2(1-\Omega(M)) .
$$

Adding these two equation together and using the fact that $\chi\left(S^{0} * M\right)=$ $2-\chi(M)$ gives the desired formula for $\Omega(M)$.

\section{Examples and remarks.}

1. We have $\Omega\left(S^{n}\right)=1$ and $\Omega(p)=1 / 2$ when $p$ is a single point.

2. A convex spherical polygon has outer angle $\Omega(M)=1 / 2+|\partial M| / 4 \pi$.

3. If $M$ is a spherical cone manifold homeomorphic to $S^{2}$, then $\Omega(M)=1$ no matter what the cone angles are. More general, any closed spherical cone manifold of dimension two has outer angle $\Omega(M)=\chi(M) / 2$. Conceptually, this follows from (3.2) and the fact that a random slice of $M$ is a closed 1-manifold of Euler characteristic zero. (Cone manifolds will be discussed systematically in $\S 5$.)

4. The 3 -sphere can be given the structure of a spherical cone manifold $M$ in such a way that its singular locus is the Hopf link, with components of length $2 \pi \alpha$ and $2 \pi \beta$. This cone manifold satisfies $\operatorname{vol}(M)=\alpha \beta$ and $\Omega(M)=\alpha+\beta-\alpha \beta$.

5. The formula in Theorem 3.1 can be iterated to yield:

$$
\Omega(M)=\sum \theta_{\alpha_{1}}\left(-\theta_{\alpha_{1} \alpha_{2}}\right) \cdots\left(-\theta_{\alpha_{s-1} \alpha_{s}}\right),
$$

where the sum is over all multi-indices $\left(\alpha_{1}<\alpha_{2}<\cdots<\alpha_{s}\right), s \geq 1$.

\section{Riemannian complexes}

In this section we define Riemannian complexes and establish the following Gauss-Bonnet formula.

Theorem 4.1 Let $M$ be a Riemannian complex with totally geodesic cells. Then

$$
\sum_{|\alpha|=\operatorname{dim}(M)} \int_{M_{\alpha}} \Psi(x) d v(x)=\sum_{\alpha}(-1)^{|\alpha|} \Theta_{\alpha} .
$$


This formula applies in particular to spherical, Euclidean and hyperbolic complexes. It relates the volume of $M$ to its Euler characteristic, defined as a weighted sum of simplices. The weight $\Theta_{\alpha}$, discussed below, is the normalized solid angle of $M$ along $M_{\alpha}$.

Smooth complexes. A Riemannian complex $M$ is a cell complex built out of Riemannian polyhedra, in the same way that a spherical complex is built out of spherical polyhedra (§3). We require that faces of cells are also cells, as in (3.1). The faces in a Riemannian complex, however, need not be totally geodesic.

The definitions of the polyhedron $N_{\alpha \beta}(x)$, the normal complex $N_{\alpha}(x)$, the inner angle $\theta_{\alpha \beta}(x)$ and the outer angle $\Omega_{\alpha}(x)$ generalize immediately to this setting.

Gauss-Bonnet. By rearranging terms as in the proof of Theorem 3.3, we obtain:

Lemma 4.2 The Euler characteristic of any compact Riemannian complex satisfies $\chi(M)=\sum_{\alpha}(-1)^{|\alpha|} \int_{M_{\alpha}} \Phi_{\alpha}(x) d v(x)$, where

$$
\Phi_{\alpha}(x)=\Psi(x)+\sum_{\beta>\alpha}(-1)^{|\beta|-|\alpha|} \int_{N_{\alpha \beta}(x)^{*}} \Psi(x, \xi) d \xi .
$$

This formula has the advantage that it shifts the focus from the boundary of $M_{\alpha}$ (coming from the lower dimension cells) to its normal bundle (coming from higher dimensional cells).

Solid angles. Let $n=\operatorname{dim}(M)$. The solid angle of $M$ at $x \in M_{\alpha}$ is defined by $\Theta_{\alpha}(x)=1$ if $|\alpha|=n$ and by

$$
\Theta_{\alpha}(x)=\sum_{\beta>\alpha,|\beta|=n} \theta_{\alpha \beta}(x)
$$

otherwise. It can be expressed more geometrically as

$$
\Theta_{\alpha}(x)=\lim _{r \rightarrow 0} \frac{\operatorname{vol}_{n}(B(x, r))}{\operatorname{vol}_{n}\left(B^{n}\right) r^{n}} .
$$

To see this, write the numerator as $\sum_{\beta} \operatorname{vol}_{n}\left(B(x, r) \cap M_{\beta}\right)$ and then interchange limit and summation.

Totally geodesic cells. The Gauss-Bonnet integral of a cell will be denoted by

$$
V_{\alpha}=\int_{M_{\alpha}} \Psi(x) d v(x)
$$


A cell $M_{\alpha}$ is totally geodesic if its second fundamental form vanishes under every inclusion $M_{\alpha} \subset \overline{M_{\beta}}$. For example, every cell in a spherical complex is totally geodesic, as is every top-dimensional cell.

Whenever $A$ and $B$ are totally geodesic submanifolds of a Riemannian manifold $C$, parallel transport along a path in $A \cap B$ preserves both $T A$ and $T B$. Thus if all cells in $M$ are totally geodesic, the angles $\theta_{\alpha \beta}(x), \Omega_{\alpha}(x)$ and $\Theta_{\alpha}(x)$ are independent of $x$. As in the spherical case, we then drop the dependence on $x$ from the notation. Moreover, we find

$$
\Phi_{\alpha}(x)=\Psi(x)\left(1-\Omega_{\alpha}\right)
$$

as in the proof of Theorem 3.3. Since $\Psi(x)=0$ unless $|\alpha|$ is even, Lemma 4.2 implies:

Theorem 4.3 A compact Riemannian complex with totally geodesic cells satisfies

$$
\chi(M)=\sum_{\alpha} V_{\alpha}\left(1-\Omega_{\alpha}\right)
$$

(Note that $V_{\alpha}=2 \theta_{\alpha}$ in the spherical case, provided $\alpha$ is even.)

Proof of Theorem 4.1. It will be useful to treat the quantities $V_{\alpha}, \Theta_{\alpha}$, $\chi_{\alpha}$ and $\bar{\chi}_{\alpha}$ as vectors, where

$$
\chi_{\alpha}=(-1)^{|\alpha|} \text { and } \bar{\chi}_{\alpha}=\chi\left(\overline{M_{\alpha}}\right) .
$$

We will also treat $\theta_{\alpha \beta}, \Omega_{\alpha \beta}$ and $L_{\alpha \beta}$ as matrices, where

$$
\Omega_{\alpha \beta}=\Omega\left(\overline{N_{\alpha \beta}}\right)=\Omega\left(\bigcup_{\alpha<\gamma \leq \beta} N_{\alpha \gamma}\right)
$$

and where

$$
L_{\alpha \beta}= \begin{cases}1 & \text { if } \alpha \leq \beta, \text { and } \\ 0 & \text { otherwise }\end{cases}
$$

(We set $\theta_{\alpha \beta}=\Omega_{\alpha \beta}=0$ unless $\alpha<\beta$.)

With this notation in place, we claim that

$$
\bar{\chi}=\chi L, \quad \Omega=\theta(L-\Omega) \text { and } \bar{\chi}=V(L-\Omega) .
$$

The first equation simply expresses the fact that

$$
\chi\left(\overline{M_{\beta}}\right)=\sum_{\alpha \leq \beta}(-1)^{|\alpha|} .
$$


The second follows from Theorem 3.1, which gives

$$
\Omega_{\alpha \beta}=\sum_{\alpha<\gamma \leq \beta} \theta_{\alpha \gamma}\left(1-\Omega_{\gamma \beta}\right)
$$

and the last follows Theorem 4.3, which gives

$$
\chi\left(\overline{M_{\beta}}\right)=\sum_{\alpha \leq \beta} V_{\alpha}\left(1-\Omega_{\alpha \beta}\right) .
$$

Now the equation $\Omega=\theta(L-\Omega)$ implies, by adding $L-\Omega$ to both sides, that $L=(I+\theta)(L-\Omega)$. Thus:

$$
V=\bar{\chi}(L-\Omega)^{-1}=\chi L(L-\Omega)^{-1}=\chi(I+\theta),
$$

or in other words

$$
V_{\beta}=\chi_{\beta}+\sum_{\alpha<\beta} \chi_{\alpha} \theta_{\alpha \beta}
$$

Summing over all $\beta$ with $|\beta|=\operatorname{dim} M$, and using the definition of $\Theta_{\alpha}$, we obtain the formula

$$
\sum_{|\beta|=n} V_{\beta}=\sum_{\alpha} \chi_{\alpha} \Theta_{\alpha}
$$

which is equivalent to the statement of the Theorem.

\section{Cone manifolds}

In this section we discuss Riemannian cone manifolds. Our treatment of their strata relies on the concept of the join $A * B$ of two spherical complexes, defined below, and the following factorization theorem.

Theorem 5.1 A compact spherical cone manifold $M$ can be canonically presented as the join $M \cong S^{a} * B$ of a sphere $S^{a}$ and a prime cone manifold $B$.

We allow the limiting cases $M \cong S^{a}$ and $M \cong B$. Here prime means there is no $C$ such that $B \cong S^{0} * C$.

Geometric cone manifolds. Let $G$ be a group acting isometrically on a complete, connected Riemannian manifold $X$ of dimension $n$. (We do not require that $G$ acts transitively.) Let $G_{p} \subset G$ denote the stabilizer of $p \in X$, 
acting on the unit tangent sphere $X_{p} \subset T_{p} X$. An $(X, G)$-manifold is a space $M$ equipped with charts into $X$ whose transition functions lie in $G$.

Following [Th], we define an $(X, G)$ cone manifold inductively as follows. For $\operatorname{dim}(X)=1$, an $(X, G)$ cone manifold is just an $(X, G)$ manifold. For $\operatorname{dim}(X)>1$, an $(X, G)$ cone manifold is a space $M$ such that each $x \in M$ has a neighborhood modeled on the cone over a compact, connected $\left(X_{p}, G_{p}\right)$ cone manifold $S_{x}(M)$, called the unit tangent cone to $M$ at $x$.

Spherical cone manifolds. A spherical cone manifold is an $\left(S^{n}, \mathrm{O}(n+1)\right)$ cone manifold. If a collection of $n$-dimensional spherical polyhedra are glued together by identifying their $(n-1)$-dimensional faces in pairs, the result is a spherical cone manifold. Every spherical cone manifold can be obtained in this way, as one can verify by induction on $n$.

Outer angles of cone manifolds. In particular, a compact spherical cone manifold $M$ can be given the structure of spherical cell complex, so its outer angle $\Omega(M)$ is can be defined as in $\S 3$. The outer angle is independent of the choice of subdivision of $M$ into spherical cells, by Proposition 3.2.

The unit tangent cone $S_{x}(M)$ of an $(X, G)$ manifold is a special case of a spherical cone manifold, since $\left(X_{p}, G_{p}\right) \cong\left(S^{n-1}, H\right)$ for some $H \subset \mathrm{O}(n)$. Of course when $H \neq \mathrm{O}(n)$, the space $S_{x}(M)$ also carries a more rigid structure.

Riemannian cone manifolds. A metric space $M$ is a Riemannian cone manifold if each $x \in M$ has a neighborhood $U$ which is isometric to an open subset of an $(X, G)$ cone manifold, for some $(X, G)$. The pair $(X, G)$ can vary from point to point.

A local model for $M$ can be constructed as follows. First, let $g$ be a metric on the unit ball $B^{n} \subset \mathbb{R}^{n}$, such that every radial arc $[0,1] \cdot x$ is a geodesic. Let $H=\mathrm{O}(n) \cap \operatorname{Isom}\left(B^{n}, g\right)$.

Let $K$ be a connected $\left(S^{n-1}, H\right)$ cone manifold. Then $K$ can be described as a spherical complex $\left(K_{\alpha}\right)$, with isometric charts

$$
\phi_{\alpha}: \overline{K_{\alpha}} \rightarrow S^{n-1}
$$

that differ on their overlaps by elements of the isometry group $H$. Since $H$ preserves $g$, the isometry type of the linear cone

$$
C_{g}\left(K_{\alpha}\right)=[0,1] \cdot \phi_{\alpha}\left(\overline{K_{\alpha}}\right) \subset B^{n}
$$

is independent of the choice of chart. These polyhedral cones can therefore be assembled to form a Riemannian cell complex $C_{g}(K)$, homeomorphic to the topological cone on $K$. 
Every point $x$ in a Riemannian cone manifold has a neighborhood $(U, x)$ isometric to $\left(C_{g}(K), 0\right)$, where $K \cong S_{x}(M), B^{n}$ is a small ball in $T_{p}(X)$, and $g$ is the pullback of the metric on $X$ under the exponential map.

When $g$ is the usual Euclidean metric on $B^{n}$, we refer to $E K=C_{g}(K)$ as the Euclidean cone over $K$.

Spherical joins. Let $A, B$ be a pair of compact spherical cone manifolds. Assume $A$ is connected unless it is isomorphic to $S^{0}$, and similarly for $B$.

The join $A * B$ is the spherical cone manifold obtained from the disjoint union of $A$ and $B$ by adding a spherical arc $[a, b]$ of length $\pi / 2$ between every pair of points $a \in A$ and $b \in B$. More formally, $A * B=S_{(0,0)}(E A \times E B)$, where $E A \times E B$ is given the product metric. For example, $S^{n} * S^{m} \cong$ $S^{n+m+1}$.

The unit tangent cones of the join are easily computed; we have

$$
S_{x}(A * B) \cong \begin{cases}\left(S_{x} A\right) * B & \text { if } x \in A \\ A *\left(S_{x} B\right) & \text { if } x \in B, \text { and } \\ S^{0} *\left(S_{a} A\right) *\left(S_{b} B\right) & \text { if } x \text { is in the open } \operatorname{arc}(a, b)\end{cases}
$$

It is convenient to adopt the convention that $S^{-1}=\emptyset$ and $A * \emptyset=A$. We say $A$ is prime if it cannot be expressed as a join $A \cong S^{0} * B$.

Proof of Theorem 5.1. If $M$ is prime we are done. Otherwise, there is least one way of writing $M \cong A * B$ with $A \cong S^{k}, k \geq 0$. Fix one such isometry, with $k$ as large as possible. We can then regard $A$ and $B$ as subsets of $M$. Clearly $B$ is prime. It remains only to show that this decomposition is unique.

Let us say $x \in M$ is a basepoint if $M \cong S^{0} * C$ with $x \in S^{0}$. Then $C \cong S_{x}(M)$ by the computation (5.1) above.

We claim that $A$ coincides with the set of all basepoints of $M$. Clearly every $x \in A$ is a basepoint. On the other hand, if $x$ is a basepoint and $x \notin A$, then either $x \in B$ or $x \in M-(A \cup B)$. In the first case, we have

$$
M \cong S^{0} * S_{x}(M) \cong S^{0} * A * S_{x} B \cong S^{k+1} * S_{x} B,
$$

by equation (5.1). But this contradicts the fact that $k$ is maximal. We obtain a similar contradiction if $x$ is in the interior of an arc $[a, b]$.

Consequently $A$ coincides with the set of basepoints of $M$. Similarly $B$ coincides with the set of points in $M$ at distance $\pi / 2$ from $A$. Thus the factors $A$ and $B$ in the join are canonically determined subspaces of $M$, and there is a unique compatible isometry $M \cong A * B$. 
Strata. Let $M$ be an $n$-dimensional Riemannian cone manifold. Its $a$ dimensional strata are the connected components of the locus

$$
M[a]=\left\{x \in M: S_{x}(M) \cong S^{a-1} * B, \text { with } B \text { prime }\right\} .
$$

Clearly $M$ is the disjoint union of the loci $M[a]$ for $0 \leq a \leq n$. We refer to the prime factor $B$ above as the normal cone to $M[a]$ at $x$; it will be denoted by $N_{x}(M)$. Thus

$$
S_{x}(M) \cong S^{a-1} * N_{x}(M)
$$

for all $x \in M[a]$.

Local models. Using the local model $U \cong C_{g}\left(S_{x}(M)\right)$ for a neighborhood of $x \in M[a]$, it is easy to verify that

$$
U \cap M[a] \cong C_{g}\left(S^{a-1}\right)
$$

indeed, $M[a] \cap U$ is isomorphic to $\left(B^{a}, g\right)$ in suitable coordinates on $B^{n}$. Thus $M[a]$ carries the structure of a Riemannian manifold of dimension $a$. The local model also shows that the geodesic rays from $x$ to nearby points $y \in M[a]$ are all contained in $M[a]$; thus $M[a]$ is totally geodesic in $M$ (equivalently, $B^{a}$ is totally geodesic in $\left(B^{n}, g\right)$ ).

All other points of $U$ belong to higher-dimensional strata, by equation (5.1). More precisely, any point $y \in U-\{x\}$ projects to a point $z \in S_{x}(M)$, and by parallel transport we have

$$
S_{y}(M) \cong S^{0} * S_{z}\left(S_{x}(M)\right) .
$$

The $S^{0}$ factor comes from the geodesic connecting $x$ to $y$. This shows:

1. The unit tangent cones $S_{y}(M)$ and $S_{x}(M)$ are isomorphic whenever $x$ and $y$ belong to the same component of $M[a]$;

2. For $b>a$, the locus $M[b] \cap U$ is just the cone over the locus $S_{x}(M)[b-1]$, with $x$ omitted; and

3. For $y \in M[b] \cap U$, we have

$$
S_{y}(M) \cong S^{a} * S_{w} N_{x}(M)
$$

where $w$ is obtained by first projecting $y$ to $S^{a-1}$ and then to $N_{x}(M)$.

The top-dimensional manifold $M[n]$ is open and dense in $M$. Our definitions imply that two-dimensional cone manifolds have only isolated singularities; more generally, $M[n-1]$ is empty, and thus $M$ is the metric completion of $M[n]$. 
Cell decompositions. Every Riemannian cone manifold $M$ admits a smooth triangulation compatible with its stratification. More precisely, $M$ can be given the structure of a Riemannian cell complex, $M=\bigcup M_{\alpha}$, in such a way that its strata are unions of cells, and each cell is a simplex.

The proof is by induction on $n=\operatorname{dim} M$, the case $n=1$ being immediate. For the inductive step, suppose $x \in M$ is modeled on the cone over $N$, $\operatorname{dim} N=n-1$. Then the cone over a triangulation of $N$ gives a triangulation of a neighborhood of $x$ in $M$. By putting these local triangulations into general position within their strata, and intersecting their cells, we obtain a decomposition of $M$ into Riemannian polyhedra. These polyhedra can then be further subdivided, by standard methods, to obtain the desired Riemannian triangulation of $M$ (see e.g. [Ca]).

Two examples. To conclude we remark that not every Riemannian cone $C_{g}(K)$ is a Riemannian cone manifold, and not every Riemannian cone manifold $M$ is an $(X, G)$ cone manifold,

To see the first statement, let $K$ be the 2-fold cover of $S^{3}$ branched over $S^{1}$. Then $K$ is an $\left(S^{3}, H\right)$ cone manifold for the trivial group $H$. Consider a metrized ball $\left(B^{4}, g\right)$ that contains no totally geodesic surfaces. Then $M=C_{g}(K)$ cannot be a cone manifold; if it were, then the cone over the branch locus $C_{g}\left(S^{1}\right)$ would be stratum of $M$, and hence totally geodesic.

For the second, consider a surface $M$ with a single irrational cone point $p$ (meaning $\Theta(p) \notin \mathbb{Q}$ ), whose metric is inhomogeneous near another point $q$. If $M$ were an $(X, G)$-manifold, then $\operatorname{Isom}(X)$ would contain a copy of $S^{1}$ coming from the closure of the group generated by holonomy around $p$; but then $M$ would admit a local Killing vector field near $q$.

\section{$6 \quad$ Euler characteristics of strata}

Let $M$ be a compact cone manifold. We say $F \subset M$ is a stratified set if $F$ is a closed union of strata of $M$.

In this section we establish the following analogue of Theorem 4.3, whose notation is explained below.

Theorem 6.1 The Euler characteristic of any stratified set $F \subset M$ is given by

$$
\chi(F)=\sum_{M^{\sigma} \subset F} V^{\sigma}\left(1-\Omega^{\sigma}(F)\right) .
$$

Corollary 6.2 The strata of $M$ satisfy $\chi\left(\overline{M^{\tau}}\right)=\sum_{\sigma \leq \tau} V^{\sigma}\left(1-\Omega^{\sigma \tau}\right)$. 
Strata and angles. The strata $M^{\sigma}$ of $M$, defined as the components of $M[r]$ for all $r \leq \operatorname{dim}(M)$, give a partition of $M$ into connected manifolds. We let $|\sigma|=\operatorname{dim}\left(M^{\sigma}\right)$, and we write $\sigma \leq \tau$ if $M^{\sigma}$ meets $\overline{M^{\tau}}$; equivalently, if $M^{\sigma} \subset \overline{M^{\tau}}$. Note that we use superscripts to index strata, just as we have used subscripts to index cells.

For $x \in M^{\sigma}$ the spherical cone manifold $N_{x}(M)$ coincides with the space of unit vectors normal to $M^{\sigma}$ at $x$. For $\tau>\sigma$, the vectors normal to $M^{\sigma}$ that point into $\overline{M^{\tau}}$ form a stratified subset of $N_{x}(M)$, which will be denoted by $N^{\sigma \tau}(x)$. This stratified set can be given the structure of a spherical complex, so its outer angle is well-defined, just as for a spherical cone manifold (see $\S 3$ ). Note that $N^{\sigma \tau}(x)$ might not be connected - e.g. the stratum $M^{\tau}$ might tend towards $M^{\sigma}$ from two different directions.

We denote the inner and outer angles of $N^{\sigma \tau}(x)$ by

$$
\theta^{\sigma \tau}(x)=\operatorname{vol}\left(N^{\sigma \tau}(x)\right) / \operatorname{vol}\left(S^{|\tau|-|\sigma|-1}\right)
$$

and

$$
\Omega^{\sigma \tau}(x)=\Omega\left(N^{\sigma \tau}(x)\right) .
$$

Using parallel transport, we find that $S_{x}(M) \cong S_{y}(M), N_{x}(M) \cong N_{y}(M)$, and $N^{\sigma \tau}(x) \cong N^{\sigma \tau}(y)$ for all $x, y \in M^{\sigma}$. Thus the angles above are independent of $x$; they will henceforth be denoted simply by $\theta^{\sigma \tau}$ and $\Omega^{\sigma \tau}$.

The solid angle of $M$ along $M^{\sigma}$ is given by

$$
\Theta^{\sigma}=\theta^{\sigma \tau}
$$

for the unique $\tau \geq \sigma$ with $|\tau|=\operatorname{dim}(M)$. (We adopt that convention that $\Theta^{\sigma}=1$ if $|\sigma|=\operatorname{dim}(M)$.) We let

$$
V^{\sigma}=\int_{M^{\sigma}} \Psi(x) d v(x)
$$

denote the intrinsic Gauss-Bonnet integral over $M^{\sigma}$.

Cells. Let $F \subset M$ be a stratified set. The normal cone to $M^{\sigma} \subset F$ at $x \in M^{\sigma}$ is defined by

$$
N^{\sigma}(F, x)=\bigcup_{\tau>\sigma, M^{\tau} \subset F} N^{\sigma \tau}(x) .
$$

It is a stratified subset of the spherical cone manifold $N_{x}(M)$. The outer angle

$$
\Omega^{\sigma}(F, x)=\Omega\left(N^{\sigma}(F, x)\right)
$$


is again independent of $x$, so it will be denoted simply by $\Omega^{\sigma}(F)$.

As discussed in $\S 5$, a cone manifold can be decomposed into Riemannian polyhedra in a manner compatible with its stratification. In particular, $F$ can be given the structure of a cell complex $\left(F_{\alpha}\right)$ in such a way that $M^{\sigma}$ is a subcomplex whenever $M^{\sigma} \subset F$. Then by Lemma 4.2 we have

$$
\chi(F)=\sum(-1)^{|\alpha|} \int_{F_{\alpha}} \Phi_{\alpha}(x) d v(x),
$$

where

$$
\Phi_{\alpha}(x)=\Psi(x)-\sum_{\beta>\alpha}(-1)^{|\beta|-|\alpha|-1} \int_{N_{\alpha \beta}(x)^{*}} \Psi(x, \xi) d \xi
$$

Fracture and repair. The decomposition $\left(F_{\alpha}\right)$ of $F$ generally includes curved cells, which fracture the totally geodesic strata in $F$. The main step in the proof of Theorem 6.1 is to show that these curved cells make no contribution to the sum (6.1) giving the value of $\chi(F)$. This type of argument is essential for applications to complex hyperbolic cone manifolds, since they have no odd-dimensional totally geodesic submanifolds (of dimension $>1$ ).

Lemma 6.3 If $x \in F_{\alpha} \subset M^{\sigma}$ with $|\alpha|<|\sigma|$, then $\Phi_{\alpha}(x)=0$.

Proof. Choose a metric $g$ on $B^{n}$ such that there is a neighborhood $U$ of $x$ in $M$ which is modeled on the cone $C_{g}\left(S_{x}(M)\right)$. Since $x \in M^{\sigma}$, we have

$$
S_{x}(M) \cong S^{|\sigma|-1} * N_{x}(M) .
$$

Since $F_{\alpha} \subset M^{\sigma}$ has codimension $|\sigma|-|\alpha|$, and $M^{\sigma} \subset F$, we have

$$
K=N_{\alpha}(x) \cong S^{d} * N^{\sigma}(F, x)
$$

where $d=|\sigma|-|\alpha|-1 \geq 0$. We will use this presentation of $K$ as a nontrivial suspension to show that $\Phi_{\alpha}(x)=0$.

Let $K_{\beta}=N_{\alpha \beta}(x)$ denote the spherical cells making up $K$, and let $C=$ $B^{n}$. Choose submanifolds with $0 \in A \subset B \subset C$ passing through $0 \in C$ which provide local models for the cells $F_{\alpha}$ and $\overline{F_{\beta}}$. Let $S_{B} \subset S_{C}$ denote the fibers of the unit normal bundle to $A$ at 0 , considered as a submanifold of $B$ and $C$ respectively. Then $K_{\beta}$ can be identified with a convex polyhedron inside $S_{B}$ and $S_{C}$. Thus it has two duals, which we will denote by $K_{\beta}^{\prime}=\left(K_{\beta}, S_{B}\right)^{*}$ and $K_{\beta}^{*}=\left(K_{\beta}, S_{C}\right)^{*}$. By the basic relation (2.2), we have

$$
\int_{N_{\alpha \beta}(x)^{*}} \Psi(x, \xi) d \xi=\int_{K_{\beta}^{\prime}} \Psi_{B}(0, \xi) d \xi=\int_{K_{\beta}^{*}} \Psi_{C}(0, \xi) d \xi
$$


Note that the holonomy group $H$ of the spherical manifold $S_{x}(M)$ acts isometrically on $\left(B^{n}, g\right)$ and trivially on $S_{x}\left(M^{\sigma}\right)$, so it also acts trivially on $S_{0}(A)$, and hence it preserves $S_{C}$. Thus the integrand $\Psi_{C}(0, \xi)$ is $H$ invariant. Since the embedding $K_{\beta} \subset S_{C}$ is well-defined up to the action of $H$, the integral of $\Psi_{C}(0, \xi)$ over $K_{\beta}^{*}$ above is also well-defined.

By Proposition 3.2, for any other subdivision of $K$ into spherical polyhedra $L_{\gamma}$ of dimension $|\gamma|$, we have

$$
\sum(-1)^{|\beta|-|\alpha|-1} K_{\beta}^{*}=\sum(-1)^{|\gamma|} L_{\gamma}^{*}
$$

as chains in $S_{C}$, modulo terms of the form $C-h(C)$ with $h \in H$ (which account for different lifts of cells to $\left.S_{C}\right)$. Since $\Psi(0, \xi)$ is $H$-invariant, it vanishes when integrated over $C-h(C)$ and we find

$$
\sum_{\beta}(-1)^{|\beta|} \int_{K_{\beta}^{*}} \Psi_{C}(0, \xi) d \xi=\sum_{\gamma}(-1)^{|\gamma|} \int_{L_{\gamma}^{*}} \Psi_{C}(0, \xi) d \xi
$$

Now by (6.3), we can write $K=S^{0} * T$ for some spherical complex $T$. Let $\left(T_{\gamma}\right)$ be a spherical cell decomposition of $T$. Then $K$ can be decomposed into cells of the form $L_{\gamma} \cong S^{0} *\left(T_{\gamma}\right)-S^{0}$, together with two 0-dimensional cells $L_{\epsilon}$ and $L_{\epsilon}^{\prime}$ that account for $S^{0}$ itself. Each cell of the form $L_{\gamma}$ contains an open geodesic joining the points of $S^{0}$; thus the dual has $L_{\gamma}^{*}$ measure zero, since it lies in the subsphere of $S_{C}$ orthogonal to $S^{0}$. On the other hand, the two remaining cells satisfy $L_{\epsilon}^{*} \cup L_{\epsilon^{\prime}}^{*}=S_{C}$. It follows that

$$
\sum_{\gamma}(-1)^{|\gamma|} \int_{L_{\gamma}^{*}} \Psi_{C}(x, \xi) d \xi=\int_{S_{C}} \Psi_{C}(x, \xi) d \xi=\Psi(x)
$$

by the basic relation (2.1). Combining this equation with equations (6.2), (6.4) and (6.5) gives $\Phi_{\alpha}(x)=0$.

Lemma 6.4 If $x \in F_{\alpha} \subset M^{\sigma}$ with $|\alpha|=|\sigma|$, then $\Phi_{\alpha}(x)=\Psi(x)\left(1-\Omega^{\sigma}(F)\right)$

Proof. In this case $F_{\alpha}$ is totally geodesic, and $N_{\alpha}(x) \cong N^{\sigma}(F, x)$. 
Proof of Theorem 6.1. By the preceding two Lemmas and equation (6.1), we have

$$
\begin{aligned}
\chi(F) & =\sum_{M_{\sigma} \subset F} \sum_{F_{\alpha} \subset M^{\sigma}}(-1)^{|\alpha|} \int_{F_{\alpha}} \Phi_{\alpha}(x) d v(x) \\
& =\sum_{M^{\sigma} \subset F}\left(1-\Omega^{\sigma}(F)\right) \int_{M^{\sigma}} \Psi(x) d v(x)=\sum V^{\sigma}\left(1-\Omega^{\sigma}(F)\right) .
\end{aligned}
$$

Here we have used the fact that $\Phi_{\alpha}(x)=0$ if $|\alpha|$ is odd, so we can ignore the factor $(-1)^{|\alpha|}$.

\section{Gauss-Bonnet for cone manifolds}

We can now complete the proof of our main result:

Theorem 7.1 For any compact cone manifold $M$ of dimension $n$, we have

$$
\int_{M[n]} \Psi(x) d v(x)=\sum_{\tau} \chi\left(M^{\tau}\right) \Theta^{\tau} .
$$

The proof will rely on:

Lemma 7.2 Let $M$ be a compact spherical cone manifold. Then the outer angle of any stratified set $F \subset M$ is given by

$$
\Omega(F)=\sum_{M^{\sigma} \subset F} \theta^{\sigma}\left(1-\Omega^{\sigma}(F)\right)
$$

where $\theta^{\sigma}=\operatorname{vol}\left(M^{\sigma}\right) / \operatorname{vol}\left(S^{|\sigma|}\right)$.

Corollary 7.3 The outer angles of the strata in any compact cone manifold $M$ satisfy

$$
\Omega^{\sigma \tau}=\sum_{\sigma<\xi \leq \tau} \theta^{\sigma \xi}\left(1-\Omega^{\xi \tau}\right)
$$

Proof. Choose $x \in M^{\sigma}$. Then $F=N^{\sigma \tau}(x)$ is a stratified subset of the spherical cone manifold $N_{x}(M)$. For each $\xi$ with $\sigma<\xi \leq \tau$, the locus $N^{\sigma \xi}(x)$ in $F$ is a union of strata with outer angle $\Omega^{\xi \tau}$ and total volume $\theta^{\sigma \xi}$. The formula then follows from the preceding result. 
Proofs of Lemma 7.2 and Theorem 7.1. The proofs for cone manifolds closely follow the proofs for Riemannian complexes. Lemma 7.2 follows from Theorem 6.1 by comparing the formulas it gives for $\chi(M)$ to $\chi\left(S^{0} * M\right)$, just as in the proof of Theorem 3.1. As for Theorem 7.1, Corollaries 6.2 and 7.3 give the matrix equations $\bar{\chi}=V(L-\Omega)$ and $\Omega=\theta(L-\Omega)$ for strata, exactly as in the proof of Theorem 4.1. The equation $\bar{\chi}=\chi L$ also holds for strata, provided we let $\chi_{\sigma}=\chi\left(\overline{M^{\sigma}}\right)-\chi\left(\partial M^{\sigma}\right)$. The same linear algebra used in the proof of Theorem 4.1 then yields Theorem 7.1 .

\section{Volumes of moduli spaces}

The traditional Gauss-Bonnet theorem implies that a closed, complex hyperbolic manifold $M \cong \mathbb{C H}^{n} / \Gamma$ satisfies

$$
\operatorname{vol}(M)=C_{n} \chi(M),
$$

where $C_{n}=(-4 \pi)^{n} /(n+1)$ !. Here the metric is normalized so that $\mathbb{C H}^{1}$ has curvature -1 . Note that $M$ is a $(G, X)$-manifold for $(G, X)=\left(\mathrm{PU}(n, 1), \mathbb{C H}^{n}\right)$.

Let $\mathcal{M}_{0, n}$ denote the moduli space of $n$ ordered points on the Riemann sphere. For $n>4$, the universal cover of $\mathcal{M}_{0, n}$ is far from being a symmetric space; in fact its automorphism group, as a complex manifold, is discrete [Roy].

Nevertheless, Thurston showed that $\mathcal{M}_{0, n}$ carries a natural complex hyperbolic metric $g_{\mu}$ for every sequence of real weights $\mu=\left(\mu_{1}, \ldots, \mu_{n}\right)$ with $0<\mu_{i}<1$ and $\sum \mu_{i}=2$. Moreover the metric completion $P(\mu)$ of $\left(\mathcal{M}_{0, n}, g_{\mu}\right)$ is a complex hyperbolic cone manifold. The $(G, X)$ cone manifold $P(\mu)$ both rigidifies and extends the original complex manifold structure on $\mathcal{M}_{0, n}$.

In this section we will use the Gauss-Bonnet theorem for cone manifolds to show:

Theorem 8.1 The complex hyperbolic volume of moduli space satisfies

$$
\frac{\operatorname{vol}\left(\mathcal{M}_{0, n}, g_{\mu}\right)}{C_{n-3}}=\sum_{\mathcal{P}}(-1)^{|\mathcal{P}|+1}(|\mathcal{P}|-3) ! \prod_{B \in \mathcal{P}} \max \left(0,1-\sum_{i \in B} \mu_{i}\right)^{|B|-1}
$$

Here $\mathcal{P}$ ranges over all partitions of the indices $(1, \ldots, n)$ into blocks $B$. We regard the quantity on the right as a formula for the cone manifold Euler characteristic $\chi(P(\mu))$. 
Shapes of polyhedra. As background for the proof, we recall that any point configuration $\left(b_{1}, \ldots, b_{n}\right)$ determines a flat metric

$$
|\omega|=\frac{|d z|}{\prod\left|z-b_{i}\right|^{\mu_{i}}}
$$

on the Riemann sphere with cone angles $2 \pi\left(1-\mu_{i}\right)$ at the points $b_{i}$. The space $(\widehat{\mathbb{C}},|\omega|)$ can be isometrically embedded as a convex polyhedron in $\mathbb{R}^{3}$; conversely, a polyhedron with $n$ marked vertices with the given cone angles determines a point configuration $\left(b_{i}\right) \in \mathcal{M}_{0, n}$. The complex hyperbolic metric $g_{\mu}$ on $\mathcal{M}_{0, n}$ can be expressed in terms of the complexified distances between the marked points $\left(b_{i}\right)$ (i.e. the periods $\int_{b_{i}}^{b_{j}} \omega$ ), which provide local coordinates on $\mathcal{M}_{0, n}$ with values in $\mathbb{C} \mathbb{H}^{n-3}$.

We also recall that the Euler characteristic of moduli space is given by $\chi\left(\mathcal{M}_{0, n}\right)=(-1)^{n-3}(n-3)$ !, as is easily proved using the fibration $\mathcal{M}_{0, n+1} \rightarrow$ $\mathcal{M}_{0, n}$.

Proof of Theorem 8.1. As shown in [Th], the metric completion $M$ of $\left(\mathcal{M}_{0, n}, g_{\mu}\right)$ is a complex hyperbolic cone manifold whose strata $M^{\mathcal{P}}$ are indexed by partitions $\mathcal{P}$ of $n$ such that $\sum_{i \in B} \mu_{i}<1$ for all $B \in \mathcal{P}$. These strata result from collisions between the vertices of the associated polyhedron which keep it locally convex. The new polyhedron has $|\mathcal{P}|$ vertices, so we have $M^{\mathcal{P}} \cong \mathcal{M}_{0,|\mathcal{P}|}$ and thus $\chi\left(M^{\mathcal{P}}\right)=(-1)^{|\mathcal{P}|+1}(|\mathcal{P}|-3)$ !. When the points in a block $B$ of $\mathcal{P}$ break up, the nearby configuration is described by $|B|$ points on a flat 2 -dimensional cone with vertex angle $2 \pi\left(1-\sum_{i \in B} \mu_{i}\right)$. Thus the solid angle of $M^{\mathcal{P}}$ in $M$ is given by

$$
\Theta^{\mathcal{P}}=\prod_{B \in \mathcal{P}}\left(1-\sum_{i \in B} \mu_{i}\right)^{|B|-1}
$$

cf. [Th, Prop 3.6(c)]. Provided $M$ is compact, the Gauss-Bonnet formula for cone manifolds then gives

$$
\operatorname{vol}(M)=C_{n-3} \sum_{\mathcal{P}} \chi\left(M^{\mathcal{P}}\right) \Theta^{\mathcal{P}}
$$

which yields the formula above. The case where $M$ is finite-volume but noncompact, which occurs only when $\sum_{i \in B} \mu_{i}=1$ for some collection of indices $B \in \mathcal{P}$, can be treated by continuity in $\left(\mu_{i}\right)$ or by using the description of cusps in $[\mathrm{Th}, \S 5]$; see also [Le]. 


\begin{tabular}{|c|c|c|c|}
\hline$q$ & $\left(p_{i}\right)$ & $\chi(P(\mu))$ & $\chi(M(\mu))$ \\
\hline 3 & $\begin{array}{lllllll} & 1 & 1 & 1 & 1 & 1 & 1\end{array}$ & $-4 / 9$ & $-1 / 1620$ \\
\hline 3 & $\begin{array}{lllll}2 & 1 & 1 & 1 & 1\end{array}$ & $1 / 3$ & $1 / 72$ \\
\hline 4 & 111111111 & $-15 / 64$ & $-1 / 172032$ \\
\hline 4 & 211111111 & $25 / 128$ & $5 / 18432$ \\
\hline 4 & $\begin{array}{llllll}3 & 1 & 1 & 1 & 1 & 1\end{array}$ & $-1 / 16$ & $-1 / 1920$ \\
\hline 4 & $\begin{array}{llllll}2 & 2 & 1 & 1 & 1 & 1\end{array}$ & $-1 / 4$ & $-1 / 192$ \\
\hline 4 & 32111 & $3 / 16$ & $1 / 32$ \\
\hline 4 & 22211 & $3 / 8$ & $1 / 32$ \\
\hline 5 & 22222 & $3 / 5$ & $1 / 200$ \\
\hline 6 & 11111111111 & $-28315 / 419904$ & $-809 / 5746705367040$ \\
\hline 6 & 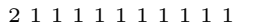 & $5663 / 93312$ & $809 / 48372940800$ \\
\hline 6 & $\begin{array}{llllllllll}3 & 1 & 1 & 1 & 1 & 1 & 1 & 1 & 1 & 1\end{array}$ & $-119 / 3888$ & $-17 / 201553920$ \\
\hline 6 & 2221111111111111 & $-287 / 4374$ & $-41 / 50388480$ \\
\hline 6 & $\begin{array}{llllllllll}4 & 1 & 1 & 1 & 1 & 1 & 1 & 1 & 1\end{array}$ & $49 / 5832$ & $7 / 33592320$ \\
\hline 6 & $\begin{array}{lllllllll}3 & 2 & 1 & 1 & 1 & 1 & 1 & 1 & 1\end{array}$ & $2107 / 46656$ & $301 / 33592320$ \\
\hline 6 & $\begin{array}{lllllllll}5 & 1 & 1 & 1 & 1 & 1 & 1 & 1\end{array}$ & $-1 / 1296$ & $-1 / 6531840$ \\
\hline 6 & 222211111111 & $637 / 7776$ & $637 / 33592320$ \\
\hline 6 & 42211111111 & $-13 / 648$ & $-13 / 466560$ \\
\hline 6 & $\begin{array}{llllllll}3 & 3 & 1 & 1 & 1 & 1 & 1 & 1\end{array}$ & $-11 / 216$ & $-11 / 311040$ \\
\hline 6 & $\begin{array}{lllllllll}3 & 2 & 2 & 1 & 1 & 1 & 1 & 1\end{array}$ & $-91 / 1296$ & $-91 / 311040$ \\
\hline 6 & 52111111 & $5 / 1296$ & $1 / 31104$ \\
\hline 6 & 433111111 & $55 / 1296$ & $11 / 31104$ \\
\hline 6 & $\begin{array}{llllllll}2 & 2 & 2 & 2 & 1 & 1 & 1 & 1\end{array}$ & $-13 / 108$ & $-13 / 62208$ \\
\hline 6 & 4221111 & $5 / 108$ & $5 / 5184$ \\
\hline 6 & $\begin{array}{llllllll}3 & 3 & 2 & 1 & 1 & 1 & 1\end{array}$ & $55 / 648$ & $55 / 31104$ \\
\hline 6 & 5331111 & $-1 / 54$ & $-1 / 1296$ \\
\hline 6 & 4411111 & $-2 / 27$ & $-1 / 648$ \\
\hline 6 & $\begin{array}{lllllll}3 & 2 & 2 & 2 & 1 & 1 & 1\end{array}$ & $55 / 432$ & $55 / 15552$ \\
\hline 6 & $\begin{array}{llllll}5 & 2 & 2 & 1 & 1 & 1\end{array}$ & $-1 / 54$ & $-1 / 648$ \\
\hline 6 & 4322111 & $-5 / 54$ & $-5 / 324$ \\
\hline 6 & $\begin{array}{llllll}3 & 3 & 3 & 1 & 1 & 1\end{array}$ & $-1 / 9$ & $-1 / 324$ \\
\hline 6 & 54111 & $1 / 12$ & $1 / 72$ \\
\hline 6 & $\begin{array}{lllllll}2 & 2 & 2 & 2 & 2 & 1\end{array}$ & $5 / 24$ & $1 / 1152$ \\
\hline 6 & 4222211 & $-1 / 9$ & $-1 / 108$ \\
\hline 6 & 332211 & $-5 / 27$ & $-5 / 216$ \\
\hline 6 & 53211 & $1 / 12$ & $1 / 24$ \\
\hline 6 & 44211 & $1 / 6$ & $1 / 24$ \\
\hline 6 & 43331 & $1 / 6$ & $1 / 24$ \\
\hline 6 & 322221 & $-5 / 18$ & $-5 / 432$ \\
\hline 6 & 52221 & $1 / 12$ & $1 / 72$ \\
\hline 6 & 43221 & $1 / 4$ & $1 / 8$ \\
\hline 6 & $\begin{array}{lllll}3 & 332 & 1\end{array}$ & $1 / 3$ & $1 / 18$ \\
\hline 6 & 33222 & $1 / 2$ & $1 / 24$ \\
\hline 8 & $\begin{array}{llllll}3 & 333 & 3 & 1\end{array}$ & $-33 / 128$ & $-11 / 5120$ \\
\hline 8 & 63331 & $9 / 64$ & $3 / 128$ \\
\hline 8 & 55222 & $9 / 32$ & $3 / 128$ \\
\hline 8 & 43333 & $9 / 16$ & $3 / 128$ \\
\hline
\end{tabular}

Table 3. Euler characteristics of the 94 orbifolds $M(\mu)$ and their cone manifold covers $P(\mu)$, with $\left(\mu_{i}\right)=\left(p_{i} / q\right)$. 


\begin{tabular}{|c|c|c|c|}
\hline$q$ & $\left(p_{i}\right)$ & $\chi(P(\mu))$ & $\chi(M(\mu))$ \\
\hline 9 & 444442 & $13 / 27$ & $13 / 648$ \\
\hline 10 & 74441 & $3 / 20$ & $1 / 40$ \\
\hline 10 & 3333332 & $293 / 1000$ & $293 / 720000$ \\
\hline 10 & 633332 & $-26 / 125$ & $-13 / 1500$ \\
\hline 10 & 93332 & $3 / 100$ & $1 / 200$ \\
\hline 10 & $\begin{array}{lllll}6 & 6 & 3 & 3 & 2\end{array}$ & $3 / 10$ & $3 / 40$ \\
\hline 10 & 533333 & $-17 / 50$ & $-17 / 6000$ \\
\hline 10 & 83333 & $3 / 25$ & $1 / 200$ \\
\hline 10 & 653333 & $39 / 100$ & $13 / 200$ \\
\hline 12 & 85551 & $7 / 48$ & $7 / 288$ \\
\hline 12 & 7722222 & $575 / 10368$ & $115 / 497664$ \\
\hline 12 & 9722222 & $-23 / 432$ & $-23 / 10368$ \\
\hline 12 & 774222 & $-23 / 216$ & $-23 / 2592$ \\
\hline 12 & 117222 & $1 / 48$ & $1 / 288$ \\
\hline 12 & 99222 & $1 / 8$ & $1 / 96$ \\
\hline 12 & 97422 & $7 / 48$ & $7 / 96$ \\
\hline 12 & 776622 & $1 / 6$ & $1 / 24$ \\
\hline 12 & 77442 & $7 / 24$ & $7 / 96$ \\
\hline 12 & 753333 & $-31 / 144$ & $-31 / 3456$ \\
\hline 12 & 555333 & $-23 / 72$ & $-23 / 2592$ \\
\hline 12 & 105333 & $1 / 12$ & $1 / 72$ \\
\hline 12 & 87333 & $13 / 48$ & $13 / 288$ \\
\hline 12 & 85533 & $7 / 24$ & $7 / 96$ \\
\hline 12 & 765333 & $17 / 48$ & $17 / 96$ \\
\hline 12 & 65553 & $1 / 2$ & $1 / 12$ \\
\hline 12 & 75444 & $11 / 24$ & $11 / 144$ \\
\hline 12 & 65544 & $13 / 24$ & $13 / 96$ \\
\hline 12 & 55554 & $7 / 12$ & $7 / 288$ \\
\hline 14 & 115552 & $6 / 49$ & $1 / 49$ \\
\hline 14 & 85555 & $24 / 49$ & $1 / 49$ \\
\hline 15 & 86664 & $37 / 75$ & $37 / 450$ \\
\hline 18 & 1188881 & $13 / 108$ & $13 / 648$ \\
\hline 18 & 137772 & $4 / 27$ & $2 / 81$ \\
\hline 18 & 1010772 & $13 / 54$ & $13 / 216$ \\
\hline 18 & 1413333 & $13 / 108$ & $13 / 648$ \\
\hline 18 & 107775 & $13 / 27$ & $13 / 162$ \\
\hline 18 & 87777 & $16 / 27$ & $2 / 81$ \\
\hline 20 & 1411555 & $99 / 400$ & $33 / 800$ \\
\hline 20 & 139666 & $69 / 200$ & $23 / 400$ \\
\hline 20 & 109966 & $99 / 200$ & $99 / 800$ \\
\hline 24 & 1917444 & $11 / 96$ & $11 / 576$ \\
\hline 24 & 149997 & $11 / 24$ & $11 / 144$ \\
\hline 30 & 2619555 & $4 / 75$ & $2 / 225$ \\
\hline 30 & 2322555 & $37 / 300$ & $37 / 1800$ \\
\hline 30 & 2211999 & $16 / 75$ & $8 / 225$ \\
\hline 42 & 3429777 & $61 / 588$ & $61 / 3528$ \\
\hline 42 & $\begin{array}{lllll}26 & 15 & 15 & 15 & 13\end{array}$ & $61 / 147$ & $61 / 882$ \\
\hline
\end{tabular}

Table 3. (continued) 
Volumes of 94 orbifolds. The pure moduli space $P(\mu)$ has a natural quotient

$$
M(\mu)=P(\mu) / S_{n}(\mu),
$$

where $S_{n}(\mu) \subset S_{n}$ is the group of permutations satisfying $\mu_{\sigma i}=\mu_{i}$.

Deligne and Mostow showed there are exactly 94 values of $\mu$ with $n \geq 5$ such that $M(\mu)$ is a complex hyperbolic orbifold [DM1], [Mos], [Th]. The volumes of these orbifolds are given by

$$
\operatorname{vol}(M(\mu))=C_{n-3 \chi}(M(\mu)),
$$

and the values of $\chi(P(\mu))$ and $\chi(M(\mu))$, computed using Theorem 8.1, are listed in Table 3 .

\section{Hyperbolic twists and non-arithmetic groups}

We conclude by discussing an invariant of non-arithmetic groups.

Let $\mathcal{T}_{0, n}$ denote the Teichmüller space of $n$ points on the sphere, regarded as the universal cover of $\mathcal{M}_{0, n}$. Fix real weights with $0<\mu_{i}<1$ such that $\sum_{1}^{n} \mu_{i}=2$. Let $\operatorname{Mod}_{0, n}(\mu)$ denote the subgroup of finite index in the mapping-class group, analogous to $S_{n}(\mu)$, which preserves the weights $\mu_{i}$. Then the natural inclusion

$$
\mathcal{M}_{0, n} / S_{n}(\mu) \hookrightarrow M(\mu)
$$

is an isometry for the metric $g_{\mu}$. Passing to the universal cover, we obtain a locally isometric developing map

$$
\delta(\mu): \mathcal{T}_{0, n} \rightarrow \mathbb{C H}^{n-3}
$$

and a representation

$$
\rho(\mu): \operatorname{Mod}_{0, n}(\mu) \rightarrow \Gamma(\mu) \subset \operatorname{Isom} \mathbb{C H} \mathbb{H}^{n-3}
$$

satisfying

$$
\delta(\mu) \circ g=\rho(g) \circ \delta(\mu) .
$$

Now suppose $M(\mu)$ is an orbifold, defined by rational weights $\left(\mu_{i}\right)=$ $\left(p_{i} / q\right)$ with $1=\operatorname{gcd}\left(p_{1}, \ldots, p_{n}, q\right)$. Let $\nu=\left(k p_{i} / q \bmod 1\right)$, where $1<k<q$ is relatively prime to $q$. We say $\nu$ is a hyperbolic twist of $\mu$ if $\sum \nu_{i}=2$. 
Theorem 9.1 If $\nu$ is a hyperbolic twist of the orbifold data $\mu$, then there is a unique holomorphic map $F$ making the diagram

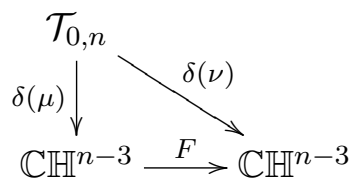

commute, and there is a unique isomorphism $\alpha: \Gamma(\mu) \rightarrow \Gamma(\nu)$ such that $F \circ g=\alpha(g) \circ F$ for all $g \in \Gamma(\mu)$.

Proof. The normal subgroup $\operatorname{Ker} \rho(\mu) \subset \operatorname{Mod}_{0, n}(\mu)$ is generated by relations coming from the complex codimension one strata of $M(\mu)$. Namely, if $\tau \in \operatorname{Mod}_{0, n}(\mu)$ is a Dehn twist around a simple loop enclosing just $b_{i}$ and $b_{j}$, and $\mu_{i}+\mu_{j}<1$ with $\mu_{i} \neq \mu_{j}$, then we must include the relation $\tau^{m_{i j}}=1$, where $m_{i j}=q / \operatorname{gcd}\left(p_{i}+p_{j}, q\right)$, to account for the cone angle of $2 \pi / m_{i j}$ along the stratum where $b_{i}$ and $b_{j}$ collide. If $\mu_{i}=\mu_{j}$, then we must add the relation $\left(\tau^{1 / 2}\right)^{m_{i j}}=1$, where $\tau^{1 / 2}$ is a half Dehn twist exchanging $b_{i}$ and $b_{j}$. Since $\operatorname{gcd}(k, q)=1$, the value of $m_{i j}$ is the same for $\left(\nu_{i}\right)=\left(k p_{i} / q\right)$ as for $\left(\mu_{i}\right)$. Thus $H=\operatorname{Ker} \rho(\mu)=\operatorname{Ker} \rho(\nu)$.

It follows that the developing map $\delta(\nu): \mathcal{T}_{0, n} \rightarrow \mathbb{C} \mathbb{H}^{n-3}$ descends to an open holomorphic map $F: X \rightarrow \mathbb{C H}^{n-3}$, where $X=\mathcal{T}_{0, n} / H$. Similarly, since $M(\mu)$ is an orbifold, $\delta(\mu)$ descends to provide an embedding of $X$ into $\mathbb{C} \mathbb{H}^{n-3}$. Indeed, $X \subset \mathbb{C} \mathbb{H}^{n-3}$ is nothing more than the preimage of $\mathcal{M}_{0, n} / S_{n}(\mu)$ inside the universal cover of $M(\mu)$. The locus $\mathbb{C} \mathbb{H}^{n-3}-X$ is a locally finite configuration of complex hyperplanes, covering the lowerdimensional strata of $M(\mu)$. By the removability of singularities for bounded analytic functions, $F$ extends to all of $\mathbb{C} \mathbb{H}^{n-3}$. Since the kernels of $\rho(\mu)$ and $\rho(\nu)$ agree, there is a unique isomorphism between their images such that $\alpha \circ \rho(\mu)=\rho(\nu)$, and (9.1) implies that $F \circ g=\alpha(g) \circ F$.

We remark that $\Gamma(\nu)$ is essentially the Galois conjugate of $\Gamma(\mu)$ coming from the automorphism $\zeta_{q} \mapsto \zeta_{q}^{k}$ of $\mathbb{Q}\left(\zeta_{q}\right) / \mathbb{Q}$, where $\zeta_{q}=\exp (2 \pi i / q)$. (The matrix entries in these groups may lie in a larger field.)

Volume ratios. The volume ratio of the hyperbolic twist is defined by

$$
\rho(\mu, \nu)=\frac{\operatorname{vol} M(\nu)}{\operatorname{vol} M(\mu)}=\frac{\chi(M(\nu))}{\chi(M(\mu))}=\frac{\chi(P(\nu))}{\chi(P(\mu))} .
$$

Note that $F$ is a contraction, by the Schwarz lemma, so $\rho(\mu, \nu) \leq 1$. In the cases at hand, it can be shown that $\Gamma(\mu)$ is arithmetic iff it has no hyperbolic twist. 


\begin{tabular}{|c|l|l|}
\hline$q$ & \multicolumn{1}{|c|}{$\left(p_{i}\right)$} & $\{\rho(\mu, \nu)\}$ \\
\hline \hline 12 & 753333 & $1 / 93$ \\
12 & 87333 & $1 / 13$ \\
12 & 655544 & $1 / 13$ \\
12 & 76533 & $1 / 17$ \\
18 & 137772 & $1 / 16$ \\
18 & 87777 & $1 / 16$ \\
20 & 1411555 & $1 / 33,4 / 33$ \\
20 & 109966 & $1 / 33,4 / 33$ \\
20 & 139666 & $1 / 46$ \\
12 & 75444 & $1 / 22$ \\
24 & 1917444 & $1 / 22$ \\
24 & 149997 & $1 / 22$ \\
15 & 86664 & $1 / 37,4 / 37$ \\
30 & 2322555 & $1 / 37,4 / 37$ \\
42 & 3429777 & $1 / 61,4 / 61$ \\
42 & 2615151513 & $1 / 61,4 / 61$ \\
\hline
\end{tabular}

Table 4. Nonarithmetic groups $\Gamma(\mu)$, with $\left(\mu_{i}\right)=\left(p_{i} / q\right)$. These 16 groups fall into 10 commensurability classes, which are almost all distinguished by the volume ratios given in the right column. (The group $\Gamma(\mu)$ for $\mu=(7,5,4,4,4) / 12$ is in a class by itself.) 
Commensurability. For 16 of the 94 orbifolds listed in Table 3, the group $\Gamma(\mu)$ is non-arithmetic. The volume ratios for these groups, computed using Theorem 8.1, are listed in Table 4.

As shown in [KM, §5], the set of volume ratios is a commensurability invariant for the group $\Gamma(\mu)$. This comes from the fact that the Euler characteristic multiplies by the degree when one passes to a finite cover, and that the boundary values of $F$ are uniquely determined by the isomorphism $\Gamma(\mu) \cong \Gamma(\nu)$. Thus the 16 groups listed fall into at least 9 commensurability classes. In fact, using previous work of Deligne-Mostow and Sauter one finds that the number of classes is exactly 10 . The group $\Gamma(\mu)$ with $\mu=$ $(7,5,4,4,4) / 12$ is not commensurable to the two other groups $\rho(\mu, \nu)=$ $1 / 22$; its trace field is $\mathbb{Q}(\cos \pi / 6)$, while the other two groups have trace field $\mathbb{Q}(\cos \pi / 12)$, For more details, see [Sau], [DM2] and [KM, $\S \S 6-7]$. 
Example: the octagon map. The orbifold weights $\mu=(3,3,5,5) / 8$ have a hyperbolic twist $\nu=(1,1,7,7) / 8$, with volume ratio

$$
\rho(\mu, \nu)=\frac{\chi(P(\nu))}{\chi(P(\mu)}=\frac{-1 / 4}{-3 / 4}=\frac{1}{3} .
$$

In fact $P(\mu)$ is the $(4, \infty, \infty)$ orbifold, while $P(\nu)$ is a cone manifold of type $(4 / 3, \infty, \infty)$; it has two cusps and a single cone point with angle $3 \pi / 2$.

The map $F: \mathbb{H} \rightarrow \mathbb{H}$ intertwining $\Gamma(\mu)$ and $\Gamma(\nu)$ is shown in Figure 5. It can be constructed by first mapping a regular ideal hyperbolic octagon to a regular ideal star, and then developing by Schwarz reflection. The map $F$ has a critical point of order 2 at the center of the picture, which expands the angle of a fundamental triangle from $\pi / 4$ to $3 \pi / 4$. The volume ratio $\rho(\mu, \nu)$ gives the average of $\|D F\|^{2}$ over the octagon, with the norm measured in the hyperbolic metric.

The value $\rho(\mu, \nu)=1 / 3$ can also be recognized as the second Lyapunov exponent of the Teichmüller curve $W_{8} \subset \mathcal{M}_{2}$ associated to billiards in the regular Euclidean octagon. Indeed, $W_{8}$ is isomorphic to $P(\mu)$, and $F$ gives the natural embedding of $W_{8}$ into the Hilbert modular surface $(\mathbb{H} \times \mathbb{H}) / \mathrm{SL}_{2} \mathbb{Z}[\sqrt{2}]$. The Lyapunov exponent has a dynamical meaning: along any infinite billiard path in the regular octagon, the number of bounces off a given side by time $T$ satisfies

$$
N(T)=a T+b T^{1 / 3}+O(1) .
$$

For a related discussion of billiards in the regular pentagon, see [Mc1, §10]. Bainbridge has shown that, more generally, any $\mathrm{SL}_{2}(\mathbb{R})$-invariant ergodic measure on the moduli space of 1 -forms $\Omega_{1} \mathcal{M}_{g}(2)$ has Lyapunov exponent $1 / 3$ [Ba1] (for background, see [Ko2]).

In view of these examples and Theorem 9.1, the non-arithmetic orbifolds $M(\mu)$ with $n>4$ can be regarded as certain higher-dimensional versions of Teichmüller curves. For more on this perspective, see [Mc2, §10] and [KM].

Example: the braid group $\boldsymbol{B}_{\mathbf{4}}$. The weights $\mu=(7,7,7,7,8) / 18$ are particular interesting, since in this case $S_{n}(\mu)=S_{4}$ and the map

$$
\rho(\mu): \operatorname{Mod}_{0,5}(\mu) \rightarrow \text { Isom } \mathbb{C H} \mathbb{H}^{2}
$$

provides a unitary representation of the braid group $B_{4}$. (More general representations of the braid group via periods are discussed in [Mc2].)

In this case the stratum $P(\mu)[1]$ has 10 components of two different types: four with the weights $(7,7,7,15) / 18$ after 2 vertices have collided, and 6 

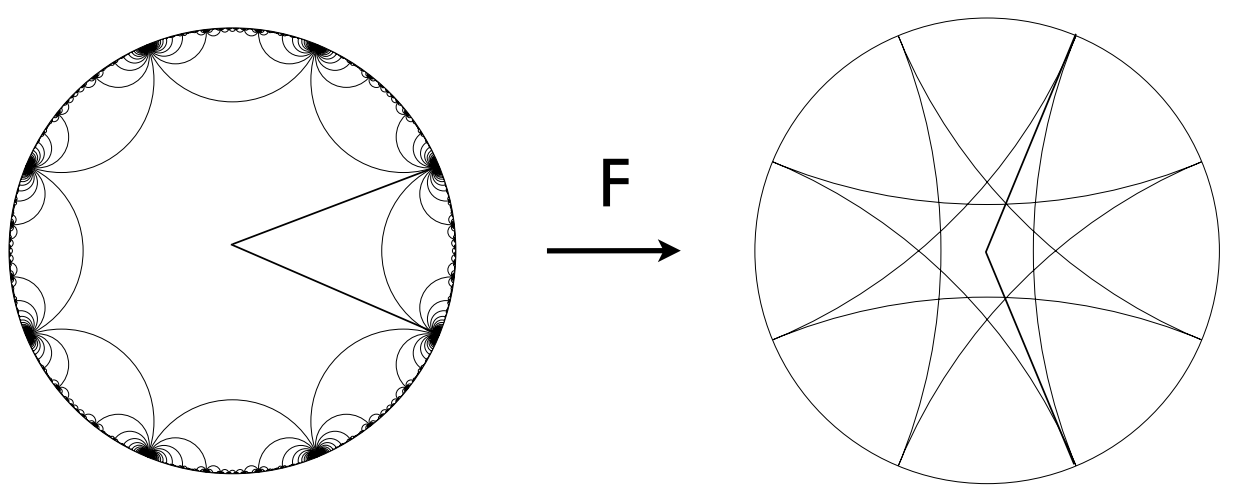

Figure 5. The hyperbolic octagon and an 8-pointed star.

with the weights $(7,7,8,14) / 18$. Similarly $P(\mu)[0]$ consists of 15 points of two different types; 12 from the weights $(7,14,15)$ and 3 from $(8,14,14)$. Using Theorem 8.1, we find

$$
\chi(P(\mu))=2-\left[4 \times \frac{1}{6}+6 \times \frac{2}{9}\right]+\left[12 \times \frac{1}{27}+3 \times \frac{4}{81}\right]=\frac{16}{27} .
$$

These weights have a unique hyperbolic twist, namely

$$
\left(\nu_{i}\right)=\left(11 \mu_{i} \bmod 1\right)=(5,5,5,5,16) / 20,
$$

and the corresponding volume ratio is given by

$$
\rho(\mu, \nu)=\operatorname{vol} \chi(P(\nu)) \chi(P(\mu))=\frac{1 / 27}{16 / 27}=\frac{1}{16} .
$$

The volume of the orbifold itself is given by

$$
\operatorname{vol}(M(\mu))=C_{2} \frac{\chi(P(\mu))}{4 !}=\frac{16 \pi^{2}}{243} .
$$

\section{References}

[AW] C. B. Allendoerfer and A. Weil. The Gauss-Bonnet theorem for Riemannian polyhedra. Trans. Amer. Math. Soc. 53(1943), 101129.

[AB] M Atiyah and C. LeBrun. Curvature, cones and characteristic numbers. Math. Proc. Cambridge Philos. Soc. 155(2013), 13-37. 
[Ba1] M. Bainbridge. Euler characteristics of Teichmüller curves in genus two. Geom. Topol. 11(2007), 1887-2073.

[Ba2] T. Banchoff. Critical points and curvature for embedded polyhedra. J. Differential Geometry 1(1967), 245-256.

[Br] K. Bromberg. Hyperbolic cone-manifolds, short geodesics, and Schwarzian derivatives. J. Amer. Math. Soc. 17(2004), 783-826.

[Ca] S. S. Cairns. A simple triangulation method for smooth manifolds. Bull. Amer. Math. Soc. 67(1961), 389-390.

[Cg] J. Cheeger. Spectral geometry of singular Riemannian spaces. J. Diff. Geom. 18(1987), 575-657.

[Cn] S. S. Chern. A simple intrinsic proof of the Gauss-Bonnet formula for closed Riemannian manifold. Ann. Math. 45(1944), 747-782.

[CHK] D. Cooper, C. D. Hodgson, and S. P. Kerckhoff. Three-Dimensional Orbifolds and Cone-Manifolds. Mathematical Society of Japan, 2000.

[DM1] P. Deligne and G. D. Mostow. Monodromy of hypergeometric functions and non-lattice integral monodromy. Publ. Math. IHES 63(1986), 5-89.

[DM2] P. Deligne and G. D. Mostow. Commensurabilities among Lattices in PU $(1, n)$. Annals of Math. Studies. Princeton Univ. Press, 1993.

[ES] V. Emery and M. Stover. Covolumes of nonuniform lattices in PU(n,1). Amer. J. Math. 135(2013), 1-22.

[FST] B. Fedosov, B-W. Schulze, and N. Tarkhanov. The index of elliptic operators on manifolds with conical points. Selecta Math. 5(1999), $467-506$.

[Ge] E. Getzler. Operads and moduli spaces of genus 0 Riemann surfaces. In The Moduli Space of Curves, pages 199-230. Birkhäuser Boston, 1995.

[Gr] A. Gray. Tubes. Birkhäuser, 2004.

[HMM] A. Hassell, R. Mazzeo, and R. B. Melrose. A signature formula for manifolds with corners of codimension two. Topology 36(1997), $1055-1075$. 
[Ha] B. Hassett. Moduli spaces of weighted pointed stable curves. Adv. Math. 173(2003), 316-352.

[KM] A. Kappes and M. Möller. Lyapunov spectrum of ball quotients with applications to commensurability questions. Preprint, 2012.

[Ko1] S. Kojima. Deformations of hyperbolic 3-cone-manifolds. J. Differential Geom. 49(1998), 469-516.

[Ko2] M. Kontsevich. Lyapunov exponents and Hodge theory. In The Mathematical Beauty of Physics (Saclay, 1996), pages 318-332. World Sci. Publishing, 1997.

[Le] E. Leuzinger. On the Gauss-Bonnet formula for locally symmetric spaces of noncompact type. Enseign. Math. 42(1996), 201-214.

[Mc1] C. McMullen. Billiards and Teichmüller curves on Hilbert modular surfaces. J. Amer. Math. Soc. 16(2003), 857-885.

[Mc2] C. McMullen. Braid groups and Hodge theory. Math. Ann., 355(2013), 893-946.

[Mc3] C. McMullen. Moduli spaces in genus zero and inversion of power series. Enseign. Math. 60(2014), 25-30.

[Mos] G. D. Mostow. Generalized Picard lattices arising from half-integral conditions. Publ. Math. IHES 63(1986), 91-106.

[Par] J. R. Parker. Complex hyperbolic lattices. In Discrete Groups and Geometric Structures, pages 1-42. Amer. Math. Soc., 2009.

[Pf] M. J. Pflaum. Analytic and geometric study of stratified spaces, volume 1768 of Lecture Notes in Mathematics. Springer-Verlag, 2001.

[Roy] H. L. Royden. Automorphisms and isometries of Teichmüller space. In Advances in the Theory of Riemann Surfaces, pages 369-384. Princeton University Press, 1971.

[Sat] I. Satake. The Gauss-Bonnet theorem for $V$-manifolds. J. Math. Soc. Japan 9(1957), 464-492.

[Sau] J. K. Sauter. Isomorphisms among monodromy groups and applications to lattices in PU(1,2). Pacific J. Math. 146(1990), 331-384. 
[Th] W. P. Thurston. Shapes of polyhedra and triangulations of the sphere. In The Epstein Birthday Schrift, volume 1 of Geom. Topol. Monogr., pages 511-549. Geom. Topol. Publ., 1998.

[Tr] M. Troyanov. On the moduli space of singular euclidean surfaces. In A. Papadopoulos, editor, Handbook of Teichmüller Theory, volume I, pages 507-540. Eur. Math. Soc., 2007.

[We] H. Weyl. On the volumes of tubes. Amer. J. Math. 61(1939), 461472.

[Yo] M. Yoshida. Volume formula for certain discrete reflection groups in PU(2,1). Mem. Fac. Sci. Kyushu Univ. Ser. A 36(1982), 1-11.

[Zo] P. Zograf. The Weil-Petersson volume of the moduli space of punctured spheres. In Mapping class groups and moduli spaces of Riemann surfaces, volume 150 of Contemp. Math., pages 367-372. Amer. Math. Soc., 1993.

\author{
Mathematics Department \\ HARVARD UNIVERSITY \\ 1 OXFORD ST \\ Cambridge, MA 02138-2901
}

